\title{
Dynamics of the human stomach
}

\author{
R. Miftahof ${ }^{1} \&$ N. Akhmadeev ${ }^{2}$ \\ ${ }^{1}$ College of Medicine and Medical Sciences, AGU, Bahrain \\ ${ }^{2}$ Kazan Medical University, Russia
}

\begin{abstract}
A mathematical model of the stomach as a soft electromyogenic biological shell is developed, which is based on detailed biological data of the structure and function of the organ. The dynamics of the spread of the wave of depolarization and concomitant stress-strain changes in the smooth muscle syncytia of the stomach were analyzed. Numerical results revealed that the fundus, the body and the antrum of the organ always experience biaxial stress-strain states, while the cardia and the pylorus undergo uniaxial stretching with crease development. The circular smooth muscle layer generated greater total forces throughout the dynamic process in comparison to the longitudinal layer. The body of the organ along the lesser curvature and the cardia-fundal area was constantly overstressed. Although the theoretical results qualitatively resemble patterns of electrical and mechanical activity observed in vivo and in vitro, there is currently no affirmative experimental evidence to conduct a detailed quantitative evaluation of the results.
\end{abstract}

Keywords: human stomach, soft thin shell, biomechanics.

\section{Introduction}

Most hollow organs of the human body, including the eyeball, the esophagus, the stomach, the gallbladder, the uterus, the ureter, and the bladder, can be viewed as thin shells. Their high endurance and enormous functionality depend on biomechanical properties of the tissues they are made of and specific arrangements of internal constituents. The distinctive anatomical appearance of organs is strongly influenced by the force systems to which they are subjected and is correlated with their structural advantages: i) containment of optimal space within and outside, ii) efficiency of physiological (biomechanical) 
function, iii) a high degree of reserved strength and structural integrity, and iv) a high strength-to-weight ratio. All of the above mentioned hollow organs share these properties. Consider, for example, that the stomach is the organ of the gastrointestinal tract that is located in the left upper quadrant of the abdomen immediately below the diaphragm. Its prime role is to accommodate and digest ingested food. Even with a small wall thickness, which in normal subjects varies from 3 to $5 \mathrm{~mm}$, with the characteristic radius of the curvature of the middle surface ranging within $10 \leq R_{i} \leq 15(\mathrm{~cm})$, it is capable of holding 2-5 liters of mixed gastric content without increasing intraluminal pressure. Large distensions and extensive mechanical activity of the organ do not affect function of the adjacent structures such as the pancreas, liver, and spleen.

With the latest advancement in mathematical modeling of complex biological systems, it has become possible to develop complex models of the abdominal viscera and to gain insight into the hidden physiological mechanisms of their function [1-4]. Such models will have enormous implications for our understanding of the pathophysiology of various diseases, e.g., gastroparesis, gastric dysrhythmias, gastroesophageal reflux disease, dumping syndrome, etc, and their pharmacological treatment.

The aims of this study are: i) to model the stomach as a thin soft biological shell, and ii) to study the dynamics of the propagation of electromechanical waves within the organ under normal physiological conditions.

\section{Physiology of the stomach}

The shape of the stomach is greatly modified by changes within itself and in the surrounding viscera that no one form can be described as typical. The chief configurations are determined by: i) the amount of the stomach contents, ii) the stage of the digestive process, iii) the degree of development of the gastric musculature, and iv) the condition of the adjacent loops of the small and large intestines. The stomach is more or less concave on its right side, convex on its left. The concave border is called the lesser curvature; the convex border, the greater curvature. The region that connects the lower esophagus with the upper part of the stomach is called the cardia. The uppermost adjacent part to it is the fundus. The fundus adapts to the varying volume of ingested food and it frequently contains a gas bubble, especially after a meal. The largest part of the stomach is known simply as the body. It functions as a reservoir for ingested food and liquids. The antrum, the lowermost part of the stomach, is usually funnel-shaped, with its narrow end connecting with the pyloric region. It empties into the duodenum - the upper division of the small intestine. The pyloric portion of the stomach tends to curve to the right and slightly upward and backward and thus gives the stomach its J-shaped appearance.

The effectiveness and diversity of physiological responses of the stomach to internal and external stimuli depend on the inherent activity of smooth muscle cells, neurons, interstitial cells of Cajal, and their topographical organization in gastric tissue. Smooth muscle cells are embedded into a network of collagenous and elastin fibers and are coupled via gap junctions into three distinct syncytia 
(muscle layers). The external longitudinal muscle layer continues from the esophagus into the duodenum. The middle uniform circular layer is the strongest and completely covers the stomach. The circular fibers are best developed in the antrum and pylorus. At the pyloric end, the circular muscle layer greatly thickens to form the pyloric sphincter. The innermost oblique muscular layer is limited chiefly to the cardia-fundal regions and progressively weakens as it approaches the pylorus.

The longitudinal, oblique and circular smooth muscle syncytia react with a variety of rhythmical movements which are a result of an electrochemical coupling phenomenon. The role of pacemakers belongs to interstitial cells of Cajal, which are located within the myenteric nervous plexus and smooth muscle syncytia [5]. Contractions in the smooth muscle are initiated by myosin light chain phosphorylation via the activation of calcium calmodulin-dependent myosin light chain-kinase. The key player in the process is free cytoplasmic calcium [6].

Elastin and collagen fibers are high-molecular-weight structural proteins built in a three dimensional loosely woven network. Elastin is soft and may be stretched to $250 \%$ of the unloaded configuration. Collagen is relatively inextensible stiff reinforcing structural component, and the main load carrying element. Collagen fibers are usually undulated and they become stiff when straightened under the action of applied loads. The strength of tissues is strongly correlated with the collagen content. The uttermost tunica serosa coats the entire organ and provides its final shape.

The submucous coat and mucous membrane of the stomach consist of epithelial and glandular cells. Their role in the biomechanics of the stomach and load distribution, in particular, is negligible.

Motor propulsive activity in the stomach originates in the upper part of the body of the organ. Three types of mechanical waves are observed: i) small isolated contraction waves, and ii) peristaltic waves that slowly move from the point of origin down toward the pyloric sphincter. These types of contractions produce slight or deep indentations in the wall and serve as mixing, crushing and pumping mechanisms for the gastric contents. The third type of wave is nonpropagating in nature and is a result of the tonic simultaneous contraction of all muscle layers that are normally superimposed on small and peristaltic contractions [7].

\section{Material characteristics}

The mechanical properties of biocomposites are highly specific and depend on the organ, the topographical site, the respective function, and species and greatly influenced by environmental risk factors and age. The soft tissue that makes the wall of the stomach is characterized as transversely anisotropic non-homogenous viscoelastic biocomposite that undergoes finite strains. This has been convincingly demonstrated in quasistatic uniaxial tests on specimens collected from different regions of the organ [8]. The force-ratio of elongation $(T-\lambda)$ data indicate that the tissue is initially compliant, $1.0<\lambda \leq 1.3-1.4$, and stiffens at 
higher loads. For $1.4<\lambda \leq 2.0$ the tissue behaves nearly elastically until it breaks at $T_{\max }=1.5 \mathrm{~N} / \mathrm{cm}$. Stretching of specimens excised in the directions of the longitudinal, circumferential and oblique layers has shown properties of transverse anisotropy. The wall has lower extensibility along the orientation of longitudinal smooth muscle fibers and the highest along of the circumferential layer. Experiments under biaxial tension have shown that the shear force applied to the tissue is significantly less, $0.01 T_{\max }$, compared with the tangential force.

The nonlinear behavior of the stomach has also been studied under complex loading, i.e., a combined inflation with subsequent external local compression of the anterior surface of the organ [9]. Instantaneous intraluminal pressure, volume and stress-strain recordings indicate that in the low pressure domain $0.1 \leq P \leq 3.0$ $(\mathrm{kPa})$ the stomach experiences biaxial stress-strain states and smooth continuous deformations in all regions. In the higher pressure range $3.0<P \leq 25(\mathrm{kPa})$ creases appear in the cardia-fundal and antrum-pyloric areas.

\section{Model assumptions}

The following assumptions are made in the formulation of a mechanical model of the stomach based on the anatomical, physiological and mechanical data of its appearance and function:

1) the stomach is a closed thin soft shell of complex geometry; it satisfies the criterion of thin shells: $\max h / R_{i}<1 / 20$, where $h$ is the thickness and $R_{i}$ are the radii of the curvature of the middle surface of the shell, and the conditions of softness: the wall of stomach does not resist compression and bending, the shear stresses are negligibly small, and creases may be formed during deformation;

2) the wall of the stomach is formed of two distinct longitudinal and circular smooth muscle layers embedded into a network of elastin and collagen fibers; the layers display an orthogonal type of weaving in the undeformed configuration;

3) the biocomposite is a nonlinear viscoelastic transversely anisotropic continuum undergoing finite strains; its "passive" component depends on the mechanics of inactive smooth muscle syncytia, collagen and elastin fibers; the "active" component - the forces of contraction and relaxation, is the result of electromechanical conjugation at the level of intracellular contractile proteins; the key biochemical factor in the process of conjugation is free calcium ions;

4) muscle layers are electrically excitable biological media; the longitudinal smooth muscle layer possesses anisotropic cable electrical characteristics, while the circular layer is electrically isotropic;

5) the initial conditions assume that the stomach is in the resting unexcited state; a single pacemaker or multiple spatially distributed pacemakers are associated with interstitial cells of Cajal; their discharge rates and intensity are assumed to be known a priori; they provide excitatory signals to the system; 
6) clamped edge boundary conditions are realized at the cardiac and pyloric ends of the shell throughout simulations.

Mathematical formulation of the model is given elsewhere [10].

\section{Results}

\subsection{Inflated stomach}

The strain distribution in the wall of the bioshell inflated by intraluminal pressure $p=20 \mathrm{kPa}$ in the state of dynamic equilibrium is shown in Fig. 1. The maximal elongation of the longitudinal muscle fibers, $\lambda_{l}=1.35$, is observed in the cardiofundal region along the greater curvature of the organ. A part of the fundus bordering the body experiences the maximal biaxial extension with $\lambda_{l}=1.35, \lambda_{c}$ $=1.04$ in that area. The cardia and the body of the stomach along the lesser curvature, both undergo biaxial deformation with $\lambda_{l}=1.01, \lambda_{c}=1.24$, while the antrum-pyloric region along the greater curvature is under uniaxial strain, $\lambda_{l}=$ $0.5, \lambda_{c}=1.16$. Negative circumferential deformations are registered in the cardia and the pylorus. Thus, the fundus and the body of the stomach undergo biaxial distension and the cardia and antrum-pyloric areas are subjects to uniaxial elongation.

Analysis of the total force distribution in the bioshell demonstrates that $\max T_{l}$ $=9.3 \mathrm{mN} / \mathrm{cm}, T_{c}=14.5 \mathrm{mN} / \mathrm{cm}$ are recorded in the fundus and the body. The maximum $T_{c}=34.7 \mathrm{mN} / \mathrm{cm}$ is registered in a small area of the body along the lesser curvature of the stomach. In the antrum and the lower cardia, total forces of average intensity $T_{l}=4.1 \mathrm{mN} / \mathrm{cm}, T_{c}=14.5 \mathrm{mN} / \mathrm{cm}$ are observed. The proximal part of the cardia remains unstressed with $T_{l}=T_{c}=0$.

\subsection{Electromechanical wave activity}

Let two identical pacemaker cells be located to the longitudinal and circular smooth muscle layers in the upper body along the greater curvature of the stomach. The cells discharge simultaneously multiple impulses $(n=5)$ of amplitude $\varphi_{0}=100 \mathrm{mV}$ and duration $t_{\mathrm{d}}=0.1 \mathrm{~s}$. The activation of ion channels on the membrane of smooth muscle causes the generation of the waves of depolarization of amplitude $\varphi_{(l, c)}=65-70 \mathrm{mV}$. The velocity of the propagation of excitation varies between the syncytia and regions of the organ. Thus the wave $\varphi_{l}$ quickly spreads within the longitudinal muscle fibers to encase a narrow zone within the anterior surface of the organ $(t=0.8 \mathrm{~s})$. The wave $\varphi_{l}$ sustains short wave-length and a constant amplitude $18 \mathrm{mV}$ throughout $2.0<t<5.6 \mathrm{~s}$. The high level of depolarization is observed in the pyloric region where $\varphi_{l}=25.2 \mathrm{mV}$ is registered.

The velocity of the propagation of the wave $\varphi_{c}$ within the isotropic circular smooth muscle layer is faster compared to $\varphi_{l}$. Thus, at $t=0.8 \mathrm{~s}$ nearly a half of the anterior and posterior surfaces of the bioshell undergo even depolarization, $60<\varphi_{c}<66.5(\mathrm{mV})$. The excitation quickly extends towards the lesser curvature. 

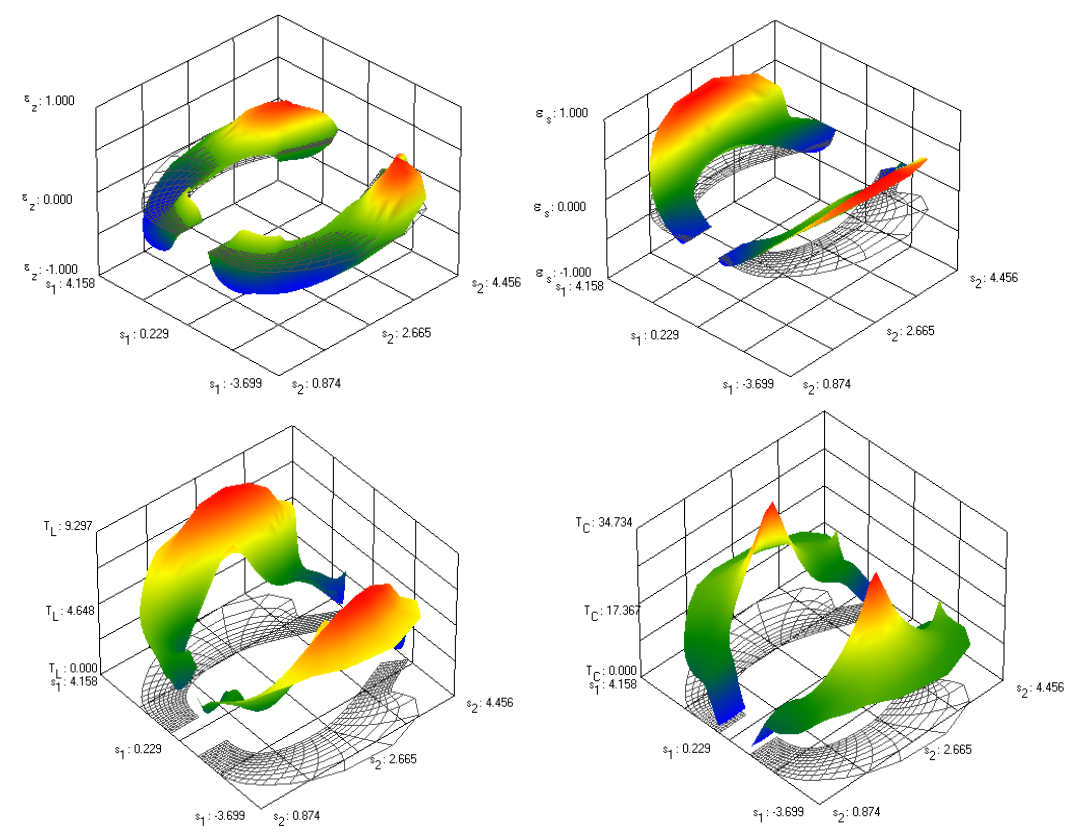

Figure 1: $\quad$ Static stress-strain distribution in the stomach.

The cardiac and pyloric regions experience extensive depolarization with a $\max \varphi_{c}=63.3 \mathrm{mV}$. At $t=5.6 \mathrm{~s}$ the longitudinal and circular smooth muscle syncytia of the stomach show a similar pattern in the distribution of depolarization with the average amplitude $\varphi_{l} \approx 3.7 \mathrm{mV}, \varphi_{c} \approx 7.5 \mathrm{mV}$.

There is a smooth distribution of active forces of contraction in the fundus and the body of the stomach where $T^{a}{ }_{l}=6.1 \mathrm{mN} / \mathrm{cm}$ and $T^{a}{ }_{c}=7.4 \mathrm{mN} / \mathrm{cm}$ are generated during $0<t \leq 5.0$ (s) (Fig. 2). The cardia and the antrum-pyloric regions generate less intense forces, $T^{a}{ }_{l}=3.0-3.6 \mathrm{mN} / \mathrm{cm}$ and $T^{a}{ }_{c}=3.7-4.4 \mathrm{mN} / \mathrm{cm}$. At $t=5.6 \mathrm{~s}$ of the dynamic process the redistribution in stresses occurs with the high level of contraction, $\max T^{a}{ }_{l}=7.9 \mathrm{mN} / \mathrm{cm}$, seen in the body of the organ. There is a concentration in active force development in the circular smooth muscle layer in the body along its lesser curvature with $\max T^{a}{ }_{c}=10.5 \mathrm{mN} / \mathrm{cm}$. The rest of the fundus and the body experience even contractions.

Analysis of deformations in the wall of the stomach shows that at $t=5.6 \mathrm{~s}$ the earlier existing zone of uniaxial stretching and wrinkling in the antrum-pyloric regions along the greater curvature has expanded to the lower part of the body. Sustained unidirectional longitudinal elongations persist in the cardia. However, a new zone of uniaxial stretch oriented along the longitudinal axis is generated along the lesser curvature of the bioshell. 

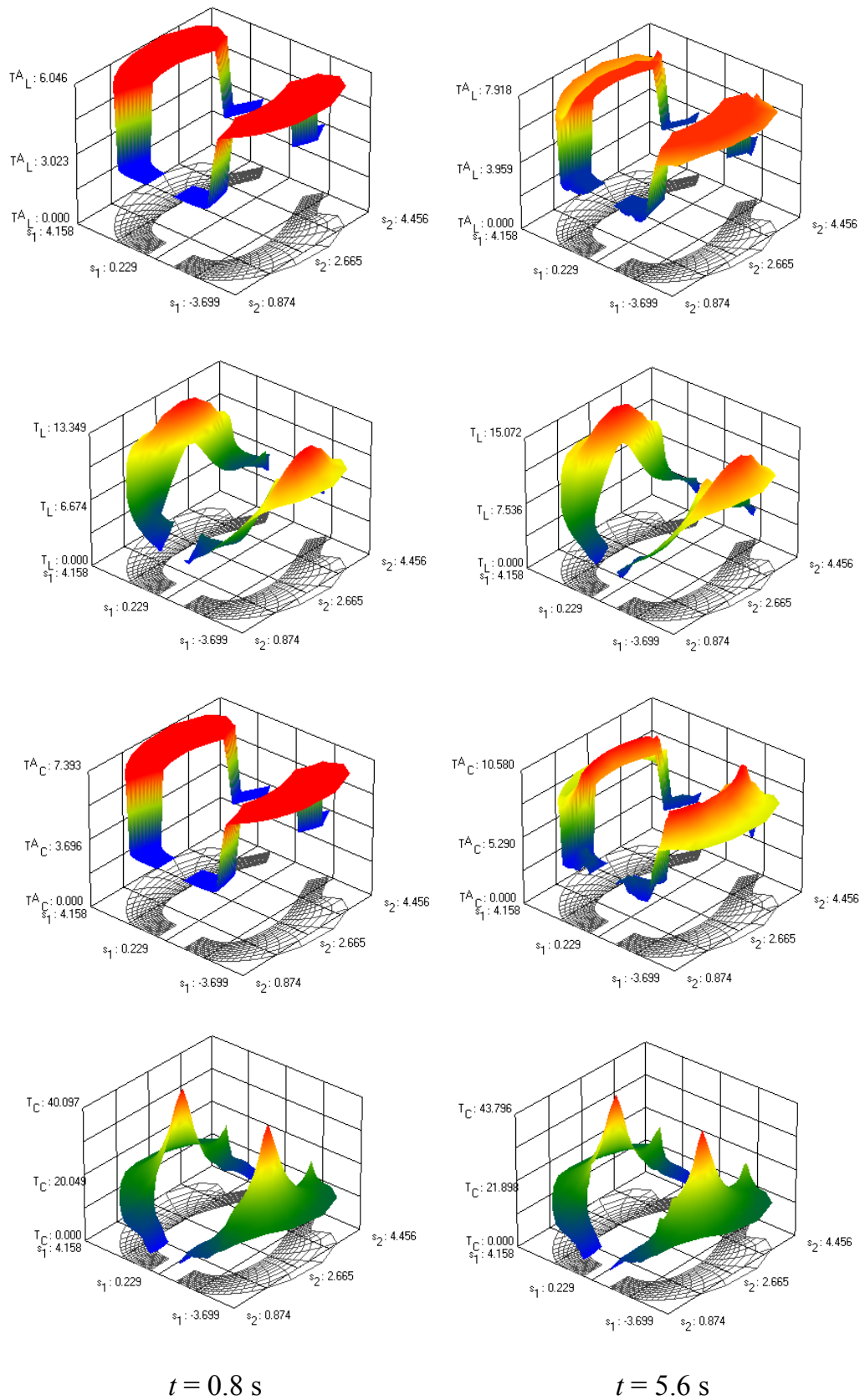

Figure 2: Dynamics of the force distribution in the stomach at times as indicated. 
The pattern of total force distribution in the shell is similar to that observed at the state of dynamic equilibrium. There is a rise in the intensity of forces, which is consistent with the process of the generation of active forces of contraction by the muscle syncytia. Thus, $\max T_{l}=15.1 \mathrm{mN} / \mathrm{cm}, T_{c}=22.4 \mathrm{mN} / \mathrm{cm}$ are recorded in the body, and $T_{l}=2.7 \mathrm{mN} / \mathrm{cm}, T_{c}=5.3 \mathrm{mN} / \mathrm{cm}$ in the cardia and the pylorus of the stomach.

\subsection{Time lag in firing of pacemakers}

We studied the effect of time delay $\left(t_{i}\right)$ in discharges of pacemaker cells on the dynamics of the force - elongation development in the bioshell. The number of impulses, their amplitude and duration correspond to the experimental conditions as described above. The pacemaker located in the longitudinal muscle syncytium fires first, followed by a discharge of the pacemaker on the circular muscle syncytium at $t_{i}=0.75 \mathrm{~s}$.

There are no significant differences in the intensity of force development. However, the delay in activation of the circular smooth muscle layer results in early wrinkling $(t=0.8 \mathrm{~s})$ in the body along the lesser curvature which persists throughout. The delayed generation of contractions in the circular smooth layer results in a reciprocal contraction-relaxation relation between the two bisyncytia. The pattern of electromechanical activity resembles peristalsis, i.e., a propagating wave of contraction - relaxation that satisfies the condition of reciprocity (Fig. 3).

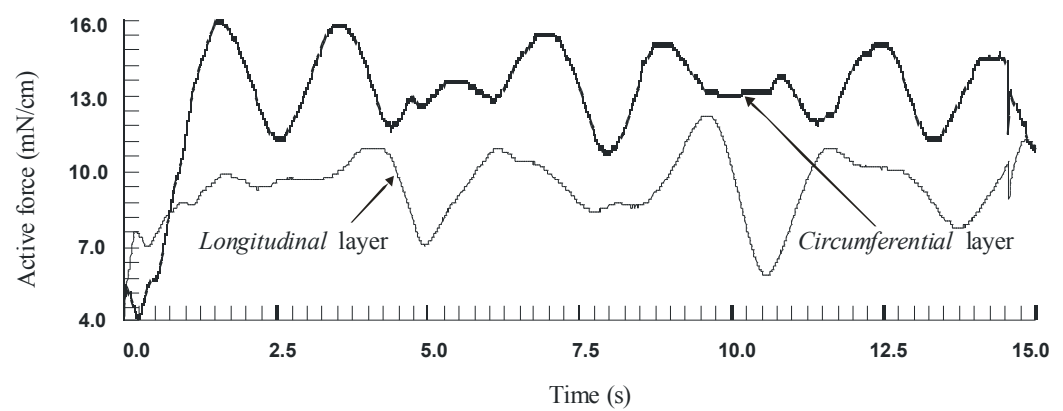

Figure 3: Changes in the active force dynamics in the longitudinal and circumferential smooth muscle layers at a "control" point in the body of the anterior surface of the human stomach.

\section{Discussion}

A first biomechanical model of the organ as a soft multilayer biocomposite shell was constructed by Miftakhov in 1983 [10]. Under general assumptions of curvilinear orthotropy and physical and geometrical nonlinearity, a mathematical formulation and numerical investigations of the dynamics of stress-strain distribution in the organ under simple and complex loadings were performed. 
The dynamics of the development of uniaxial stress-strained states in the cardia and pylorus as a function of intraluminal pressure was demonstrated computationally and supported experimentally. Results offered a valuable insight into the mechanism of blunt abdominal trauma with rupture of the anterior wall of the stomach and gave a biomechanical explanation for the Mallory-Weiss syndrome. It was thought previously that atrophic changes in the gastric mucosa and submucous layer were responsible for longitudinal tears in the cardia-fundal region and life threatening intragastric bleeding. The model study proved that the anatomical structure and configuration of the stomach per se makes these regions more susceptible, than the others, to linear submucous breaks and ruptures.

The current model is an extension of the previous model and incorporates cable electrical properties of the longitudinal and circular smooth muscle syncytia along with mechanical nonlinearity. We continue to adopt the phenomenological approach to describe stomach behavior and, thus, we exclude from considerations a detailed modeling of intricate intracellular signaling mechanisms involved in complex physiological responses of the organ. Special emphasis in this study was given to the questions of electromechanical coupling in smooth muscle syncytia and cable electromechanical wave activity.

In the current study we restricted our attention to the cable, rather than to more general oscillatory, properties of smooth muscle syncytia. The network of interstitial cells of Cajal - pacemakers of gastric motility, was substituted by a single cell or a set of spatially distributed cells. They discharged a priori known electrical impulses. Even with these constructive simplifications, the model reproduced and predicted: 1) patterns of the propagation of the wave of excitation within the electrically anisotropic longitudinal and electrically isotropic circular smooth muscle syncytia; 2) small isolated contraction waves in the two smooth muscle syncytia and non-propagating tonic simultaneous contractions of both muscle layers superimposed on small contractions; 3) the development of wrinkles in the cardia, the body along the lesser curvature and the pylorus, and 4) high levels of tension in the body of the stomach along the lesser curvature.

Care should be taken though in transferring the results of simulations to explain real biomechanics of the human stomach. One has to bear in mind that the biological plausibility of the model is constrained by the model assumptions, despite the fact that the theoretical results resemble qualitatively patterns of electrical and mechanical activity that are observed in mainly animal studies in vivo and in vitro. At the moment there is no direct affirmative experimental evidence obtained on human subjects to run a detailed quantitative evaluation and comparison of the computational data.

\section{References}

[1] Miftahof, R., Nam, H.G. \& Wingate D. L. Mathematical modeling and simulation in enteric neurobiology, World Sci., 2008 (in press)

[2] Pullan, A., Cheng, L., Yassi, R. \& Buist, M. Modeling gastrointestinal bioelectric activity. Prog. Biophys. \& Mol. Biol. 85, pp. 523-550, 2004 
[3] Cheng, L., Komuro, R., Austin, T.M., Buist, M.L. \& Pullan A. J. Anatomically realistic multiscale models of normal and abnormal gastrointestinal electrical activity. World J. Gastroenterol. 7, pp. 13781383, 2007

[4] Corrias, A. \& Buist, M.L. A quantitative model of gastric smooth muscle cellular activation. Annals Biomed. Eng. 35, pp. 1595-1607, 2007

[5] Hirst, D. G. S. \& Suzuki, H. Involvement of interstitial cells of Cajal in the control of smooth muscle excitability. J. Physiol. 576, pp. 651-652, 2006

[6] Bárány, M. Biochemistry of Smooth Muscle Contraction. Academic Press, p. 418,1996

[7] Alvarez, W.C. \& Zimmermann, A. Movements of the stomach. Am. J. Physiol. 84, pp. 261-270, 1928

[8] Miftakhov, R.N. 1981a Age changes of the 'quasi-equilibrium' module of elasticity of the human stomach. In: Shell interactions with fluids, Acad. Sci. USSR. pp. 197-204 (in Russian)

[9] Miftakhov, R.N. 1983b Experimental investigations of the stomach under complex loading. In: Hydroelasticity of shells, Acad. Sci. USSR, pp.172181 (in Russian)

[10] Miftakhov, R.N. Experimental and numerical investigations of soft shells. Ph.D. thesis. Kazan State Univ., 1983, (in Russian). 